\title{
Hematopoietic Stem Cell Transplant for the Treatment of X-MAID
}

\author{
Sarah E. Henrickson ${ }^{1,2}$, Isabelle Andre-Schmutz ${ }^{3,4}$, Chantal Lagresle-Peyrou ${ }^{3,4}$, \\ Matthew A. Deardorff ${ }^{5}$, Harumi Jyonouchi ${ }^{6}$, Benedicte Neven ${ }^{4,7}$, Nancy Bunin ${ }^{8}$ and \\ Jennifer R. Heimall ${ }^{1 *}$
}

\begin{abstract}
${ }^{1}$ Division of Allergy and Immunology, The Children's Hospital of Philadelphia, Philadelphia, PA, United States, ${ }^{2}$ Perelman School of Medicine, Institute for Immunology, University of Pennsylvania, Philadelphia, PA, United States, ${ }^{3}$ Laboratory of Human Lymphohematopoiesis, INSERM UMR 1163, Paris, France, ${ }^{4}$ Imagine Institute, Paris Descartes - Sorbonne Paris Cité University, Paris, France, ${ }^{5}$ The Children's Hospital of Philadelphia, Department of Human Genetics, Philadelphia, PA, United States, ${ }^{6}$ Division of Allergy/Immunology and Infectious Diseases, Rutgers-New Jersey Medical School, Newark, NJ, United States, ${ }^{7}$ Pediatric Immuno-Hematology Unit, Necker Children Hospital, Assistance-Publique Hopitaux de Paris, Paris, France, ${ }^{8}$ Division of Oncology, Bone Marrow Transplant and Cellular Therapy, The Children's Hospital of Philadelphia, Philadelphia, PA, United States
\end{abstract}

\section{OPEN ACCESS}

Edited by:

Ivan K. Chinn

Baylor College of Medicine,

United States

Reviewed by:

Eric J. Allenspach,

Seattle Children's Research Institute,

United States

Lyn R. Griffiths,

Queensland University of Technology,

Australia

*Correspondence:

Jennifer R. Heimal

heimallj@email.chop.edu

Specialty section:

This article was submitted to

Pediatric Immunology,

a section of the journal

Frontiers in Pediatrics

Received: 02 December 2018

Accepted: 12 April 2019

Published: 14 May 2019

Citation: Henrickson SE, Andre-Schmutz I, Lagresle-Peyrou C, Deardorff MA, Jyonouchi $H$, Neven B, Bunin N and Heimall JR (2019) Hematopoietic Stem Cell Transplant for the Treatment

of X-MAID. Front. Pediatr. 7:170. doi: 10.3389/fped.2019.00170
We report outcomes after hematopoietic stem cell transplant for three patients with X-MAID, including 1 patient from the originally described cohort and two brothers with positive TREC newborn screening for SCID who were found to have a T-B-NK+ SCID phenotype attributable to $X$-linked moesin associated immunodeficiency (X-MAID). A c.511C > T variant in moesin was identified via exome sequencing in the older of these siblings in the setting of low lymphocyte counts and poor proliferative responses consistent with SCID. He received reduced intensity conditioning due to CMV, and was transplanted with a T-depleted haploidentical (maternal) donor. His post-transplant course was complicated by hemolytic anemia, neutropenia, and sepsis. He had poor engraftment, requiring a 2nd transplant. His younger brother presented with the same clinical phenotype and was treated with umbilical cord blood transplant following myeloablative conditioning, has engrafted and is doing well. The third case also presented with severe lymphopenia in infancy, received a matched related bone marrow transplant following myeloablative conditioning, has engrafted and is doing well. These cases represent a novel manifestation of non-radiosensitive X-linked form of T-B-NK+ SCID that is able to be detected by TREC based newborn screening and effectively treated with HCT.

Keywords: SCID, HCT, WES, NBS, moesin

\section{BACKGROUND}

$\mathrm{X}$-linked moesin-associated immunodeficiency (X-MAID) is a recently described form of primary immunodeficiency, characterized by severe leukopenia affecting $\mathrm{T}$ and $\mathrm{B}$ cells as well as neutrophils (1). X-MAID is one of a group of immunodeficiencies attributed to defects in cytoskeletal regulation (2). Moesin normally functions as an anchor of the actin filaments to the plasma membrane together with the proteins ezrin and radixin. These are collectively known as the ERM family of proteins. Lymphocytes have been described to have higher expression of moesin and ezrin as compared to radixin.

Prior to the publication of the X-MAID patient descriptions, isolated deficiency of moesin in mice was reported (3). The moesin deficient mice had an overall leukopenia with more pronounced 
loss of lymphocytes, particularly CD8 cells. In other mouse models, moesin deficiency is associated with a reduced proliferative capability of CD8 Treg cells and development of autoimmune symptoms consistent with lupus $(3,4)$. In addition, the mouse model demonstrated that the moesin deficient $\mathrm{T}$ and $\mathrm{B}$ cells demonstrated impaired ability to egress from the thymus and bone marrow, respectively, and that once in the periphery, moesin deficient $\mathrm{T}$ and $\mathrm{B}$ cells were also more likely to be held in the spleen and other secondary lymphoid organs. In $\mathrm{X}$-MAID patients with the $\mathrm{c} .511 \mathrm{C}>\mathrm{T}$ missense variant, moesin expression is reduced in B cells, CD4, and CD8 cells. These patients also demonstrated an impaired proliferative response to the phytohemaglutinin mitogen stimulation. In addition, a defect in thymic egress similar to these mouse models was described in the $\mathrm{T}$ cell populations of X-MAID patients bearing mutated moesin proteins (1). In addition, these patients were also noted to have neutropenia, the etiology of which is unclear. However, the carrier mothers of patients with the c.511C > T variants were noted to have skewed lionization in the peripheral blood mononuclear cell as well as polymorphonuclear cells. This skewing was not seen in their epithelial cells.

Despite their severely low T cell counts, only one of the seven originally described X-MAID patients was treated with allogeneic hematopoietic stem cell transplantation (HCT). Other, more well-known defects of cytoskeletal regulation associated with combined immunodeficiency include Wiskott Aldrich Syndrome (WAS) and DOCK8 deficiency (2). Both of these are able to be corrected though HCT. However, in WAS it has been noted that there is a spectrum of disease, and some patients do quite well with supportive care alone. In addition, WAS patients treated with HCT require a high degree of sustained engraftment to maintain correction of their immunodeficiency and to avoid development of severe autoimmunity (5). In contrast, Severe Combined Immune Deficiency (SCID) constitutes an unquestionable and urgent indication for HCT. Several studies have highlighted better outcomes for SCID patients diagnosed and transplanted early in life, a key element of justifying implementation of newborn SCID screening in the US and other countries $(6,7)$. However, the T-B-NK+ SCID phenotype poses unique challenges for transplant, since this phenotype can commonly be associated with radiosensitive SCID genotypes caused by DCLRE1C (Artemis), LIG4, PRKDC, NHEJ1, and NBN (NBS1) variants which can be associated with a higher risk of late effects when myeloablative conditioning is used (8). Prior to the description of X-MAID, only those SCID patients with pathologic variants in RAG1 or RAG2, were non-radiosensitive forms of T-B-NK+ SCID and therefore able to better tolerate higher intensity conditioning than those with a similar phenotype due to radiosensitivity associated genetic defects.

\section{CASE PRESENTATIONS}

We report here three cases of patients with X-MAID treated with HCT due to their initial SCID clinical presentation. The two first patients are Hispanic male siblings with X-MAID born to non-consanguineous parents and treated with HCT due to their initial clinical presentation being consistent with a phenotype of T-B-NK+ SCID. Written informed consent was obtained from the parents for publication of these cases and ongoing research based study of their children's immunodeficiency. Patient 1 was first identified due to abnormally low $\mathrm{T}$ cell receptor excision circles (TREC)s on newborn screening for SCID. There was no maternal engraftment and HIV testing was negative. There was no family history of SCID or other immunodeficiency. There was one maternal half-brother but no full siblings. The physical exam at presentation was normal. Prophylaxis was initiated at 1 week of life with IVIG and sulfamethoxazole/trimethoprim sulfate and fluconazole. He was confirmed to have T-B-NK+ SCID based on profound $\mathrm{T}$ and $\mathrm{B}$ cell lymphopenia (Table 1), and a very poor response to PHA stimulation ( $2 \%$ of normal). In addition he had neutropenia with absolute neutrophil count of 200 cells/uL. A commercially available comprehensive SCID genetic testing panel demonstrated him to be a carrier of a heterozygous pathogenic variant in $R M R P$, with neither an identified second variant nor any features of Cartilage-Hair Hypoplasia (OMIM 250250). He developed persistent neutropenia by 2 months of age (Table 1).

Diarrhea developed at 2 months, and he was noted to be CMV PCR positive in blood. Therapy was initiated with valganciclovir. There was no suitable HLA-matched unrelated donor or cord blood and he emergently received a maternal haploidentical CD3/CD19-depleted HCT 2 weeks later (Cell dose: CD34 $11.6 \times 10^{6}$ cells/kg; CD3 and CD19 below level of detection). Due to a lack of identification of a known RAG1 or RAG2 variant and without results demonstrating lack of radiosensitive SCID in the setting of a T-B-NK+ phenotype, the decision was made to minimize alkylator therapy. He received reduced intensity conditioning that included thymoglobulin ( $3 \mathrm{mg} / \mathrm{kg}$ daily for three doses) and busulfan (1 $\mathrm{mg} / \mathrm{kg}$ for eight doses with pharmacokinetics, PK 1159 micromole* min/L). He became CMV PCR-negative 2 weeks after transplant. At discharge, he remained on fluconazole and pentamidine prophylaxis, IVIG, and treatment-dose foscarnet. He developed autoimmune hemolytic anemia (AIHA) at 3 months posttransplant, which was treated with methylprednisone. At 5 and 7 months post-transplant he was admitted with Enterobacter septicemia associated with his central line. He developed neutropenia at 6 months post HCT that was progressive, but responsive to G-CSF supplementation 3 days a week to maintain ANC over 500. At 16 months post HCT, his T cell engraftment was $83 \%$ and CD3 counts were normal, but TREC counts remained low, with absent myeloid engraftment, G-CSF dependent neutropenia, poor B cell counts with low IgA and IgM and continued need for immunoglobulin replacement. Bone marrow biopsy demonstrated normocellular marrow with trilineage hematopoiesis. CMV PCR remained negative throughout this period of neutropenia.

At 3 years, 2.5 months of age, he received a second MMRD HCT using TCR alpha/beta depleted PBSC from the same donor, in the setting of no other available match. Conditioning was myeloablative and consisted of fludaribine $\left(40 \mathrm{mg} / \mathrm{m}^{2}\right.$ for 4 doses), melphalan (140 mg/m² for 1 dose), thiotepa (10 mg/kg for 1 dose) and thymoglobulin ( $3 \mathrm{mg} / \mathrm{kg}$ for 3 doses) with a goal 
TABLE 1 | Laboratory values for proband prior to and after mismatched related donor HCT.

\begin{tabular}{|c|c|c|c|c|c|c|c|c|c|}
\hline & Pre-HCT & Pre-HCT & $\begin{array}{l}3 \text { months } \\
\text { post HCT }\end{array}$ & $\begin{array}{l}6 \text { months } \\
\text { post HCT }\end{array}$ & $\begin{array}{l}7.5 \text { months } \\
\text { post HCT }\end{array}$ & $\begin{array}{l}8.5 \text { months } \\
\text { post HCT }\end{array}$ & $\begin{array}{l}12 \text { months } \\
\text { post HCT }\end{array}$ & $\begin{array}{l}16 \text { months } \\
\text { post HCT }\end{array}$ & $\begin{array}{c}2 \text { years post } \\
\mathrm{HCT}\end{array}$ \\
\hline $\begin{array}{l}\text { Age of the patient at } \\
\text { analysis }\end{array}$ & DOL 5 & 2 months & 5 months & 8 months & 9.5 months & 10.5 months & 14 months & 18 months & $\begin{array}{l}\text { 27-28 } \\
\text { months }\end{array}$ \\
\hline ANC/UL & 1,600 & 200 & 4,940 & 1,020 & $396^{\star}$ & $310^{\star}$ & $320^{*}$ & $3,630^{*}$ & 670 \\
\hline ALC/UL & 200 & 536 & 810 & 156 & 382 & 640 & 1,620 & 1,650 & 1,460 \\
\hline CD3+/ul & 41 & 175 & 55 & 77 & 184 & 421 & 1,281 & 1,524 & 1,172 \\
\hline CD4+/uL & 28 & 48 & 5 & 12 & 84 & 135 & 282 & & 413 \\
\hline CD8+/uL & 11 & 123 & 36 & 50 & 81 & 241 & 847 & & 694 \\
\hline CD4+/CD45RA+ (\%) & & 5.7 & 0.4 & 2.9 & 9.5 & 10.6 & 6.2 & & 11.7 \\
\hline CD4+/CD45RO+ (\%) & & 5.4 & 0.6 & 5.7 & 10.2 & 9.9 & 10.9 & & 16.1 \\
\hline $\begin{array}{l}\text { Mitogens (PHA) \% of } \\
\text { control CPM }\end{array}$ & & $\begin{array}{c}2 \% \\
\text { CPM } 887\end{array}$ & $\begin{array}{c}<1 \% \\
\text { CPM 1,127 }\end{array}$ & & $\begin{array}{c}1 \% \\
\text { CPM 2,439 }\end{array}$ & & & & \\
\hline TRECS $^{\star \star \star}$ & & & & 1,398 & & 2,283 & & & 2,962 \\
\hline CD19+ B cells/uL & 3 & 255 & 704 & 46 & 167 & 84 & 86 & & 91 \\
\hline NK cells/uL & 78 & 110 & 51 & 32 & 36 & 141 & 245 & & 164 \\
\hline Chimerism $^{\wedge} \wedge$ & & & $82 \%$ & $26 \%$ & $25 \%$ & & $26 \%$ & $41 \%$ & \\
\hline Chimerism (CD3) & & & $57 \%$ & $36 \%$ & $76 \%$ & & $83 \%$ & $83 \%$ & \\
\hline Chimerism (CD19) & & & $96 \%$ & $49 \%$ & & & & $15 \%$ & \\
\hline Chimerism (NK) & & & $96 \%$ & $70 \%$ & & & & & \\
\hline Chimerism (Myeloid) & & & $79 \%$ & $17 \%$ & & & & $2 \%$ & \\
\hline
\end{tabular}

*, on G-CSF supplementation; ${ }^{\star * *}$, Count per $10^{6}$ CD3 cells; ^^, Unenriched whole blood.

TABLE 2 | Immune variants on proband WES.

\begin{tabular}{llccc}
\hline Gene & $\begin{array}{l}\text { Chr: position, } \\
\text { ref, alt }\end{array}$ & CADD score & $\begin{array}{c}\text { PROVEAN } \\
\text { score }\end{array}$ & $\begin{array}{c}\text { Depth of } \\
\text { coverage }\end{array}$ \\
\hline MSN & $\begin{array}{l}\mathrm{X}: 64951012 \mathrm{C}>\mathrm{T} \\
\mathrm{pR} 171 \mathrm{~W}\end{array}$ & 33 & -6.88 & $54 \mathrm{x}$ \\
PRKDC & $\begin{array}{l}\text { ch8: } 48842520 \\
\mathrm{~T}>\mathrm{C} \text { pF649L }\end{array}$ & 20.3 & -3.42 & $107 \mathrm{x}$ \\
& & & & \\
\hline
\end{tabular}

to correct his incomplete immune reconstitution, and achieve myeloid engraftment. He demonstrated recovery of neutrophil counts to $>1,000$ cells/uL by 10 days post HCT with $100 \%$ engraftment at 3 months post-transplant. He did experience reactivation of $\mathrm{CMV}$ which was resistant to treatment with foscarnet, but did respond to CMV-specific viral cytotoxic $\mathrm{T}$ cells. Unfortunately, at 3 months post-transplant he developed polyserositis of unclear etiology which manifested as pericardial and pleural effusions. His symptoms progressed to ARDS and he had acute pulmonary hemorrhage which led to his death.

Clinical exome sequencing of the proband and the parents as a trio analysis was performed 6 months after his first transplant utilizing DNA obtained pre-transplant from a skin biopsy. He was noted to have a heterozygous variant of undetermined significance (VUS) in PRKDC (c.1945T >C; Table 2). Deletion and duplication testing of $P R K D C$ identified no second variant, making it unlikely that this variant was causal for an autosomal recessive form of T-B-NK+ radiosensitive SCID. In contrast, a VUS in MSN, an X-linked gene encoding moesin (c.511 C>T; Table 2) was identified and confirmed by Sanger sequencing.
This variant was reported based on moesin-deficient murine data showing decreases in B and $\mathrm{T}$ cells (3). This variant was not observed in the ExAC dataset (exac.broadinstitute.org), where MSN also demonstrates a pLI of 0.99 and a missense constraint $\mathrm{z}$-score of 2.78 , indicating poor tolerance for loss or altered function of this gene. In addition, this variant was later reported to be associated with X-MAID.

The second affected boy, a full sibling to the proband, also presented with a presumptive positive TREC screen on NBS for SCID, with flow cytometry demonstrating a virtual absence of T, B and NK cells and poor proliferation to PHA stimulation but normal neutrophils. At 1 week of life he was started on sulfamethoxazole/trimethoprim sulfate, fluconazole prophylaxis and IVIG (Table 3). Due to the known variant in his brother, patient 2 underwent targeted Sanger sequencing for the moesin variant and he was confirmed to have the same pathogenic variant in moesin as his older sibling (Table 2). Although he did not have CMV infection, after discussion with the family and care team, it was decided that this patient would also be treated with HCT for his T-B-NK+ SCID phenotype. At 2 months, he underwent HCT with a 6/6 matched cord blood (Cell dose: CD34 $3.59 \times 10^{5}$ cells $/ \mathrm{kg}$; CD3 $3.75 \times 10^{5}$ cells $/ \mathrm{kg}$ ) with conditioning that included busulfan $(0.8 \mathrm{mg} / \mathrm{kg}$ for 16 doses with pharmacokinetics for AUC: PK 1526 micromole*min/L), fludarabine $(1.33 \mathrm{mg} / \mathrm{kg}$ for 4 doses), and thymoglobulin (3 $\mathrm{mg} / \mathrm{kg}$ daily for 3 doses). He was also found to have congenital hypothyroidism on NBS and was started on levothyroxine.

Similarly to his older brother, the post-transplant course was complicated by AIHA, and treated with rituximab and prednisone. He had limited skin graft vs. host disease (GVHD) and was treated with sirolimus. Post HCT infections included 
TABLE 3 | Laboratory values for younger sibling prior to and after matched cord blood HCT.

\begin{tabular}{|c|c|c|c|c|c|}
\hline & Pre-HCT & $\begin{array}{c}39 \text { days post } \\
\text { HCT }\end{array}$ & $\begin{array}{c}81 \text { days post } \\
\text { HCT }\end{array}$ & 1 year post $\mathrm{HCT}$ & 2 years post $\mathrm{HCT}$ \\
\hline $\begin{array}{l}\text { Age of the patient at } \\
\text { analysis }\end{array}$ & DOL7 & 3.5 months of age & 5 months of age & 13 months of age & 26 months of age \\
\hline ANC/UL & 2,020 & 4,620 & 5,160 & 10,320 & 1,630 \\
\hline ALC/UL & 170 & 600 & 1,320 & 450 & 3,210 \\
\hline $\mathrm{CD} 3+/ \mathrm{ul}$ & 136 & 232 & 182 & 550 & 2,087 \\
\hline CD4+/uL & 103 & & 164 & 277 & 1,197 \\
\hline CD8+/uL & 33 & & 11 & 181 & 745 \\
\hline CD4+/CD45RA+ (\%) & 39.4 & & 2 & 6.2 & 28.4 \\
\hline CD4+/CD45RO+ (\%) & 16.7 & & 12.4 & 29.8 & 8.4 \\
\hline Mitogens (PHA) & $2 \%$ & & $4 \%$ & & $100 \%$ \\
\hline$\%$ of control CPM & CPM 5,938 & & CPM 7,747 & & CPM 171,606 \\
\hline TRECS $^{\star \star \star}$ & & & $\begin{array}{c}148 \\
(1,484-5,327)\end{array}$ & $\begin{array}{c}4,578 \\
(>6,794)\end{array}$ & $\begin{array}{c}25,195 \\
(>6,794)\end{array}$ \\
\hline CD19+ B cells/uL & 10 & & 558 & 0 & 870 \\
\hline NK cells/uL & 24 & & & 213 & 173 \\
\hline Chimerism $^{\wedge} \wedge$ & & $100 \%$ & $100 \%$ & $100 \%$ & $100 \%$ \\
\hline Chimerism (CD3) & & QNS & QNS & $100 \%$ & $100 \%$ \\
\hline Chimerism (CD19) & & QNS & QNS & $100 \%$ & $100 \%$ \\
\hline Chimerism (NK) & & $100 \%$ & $100 \%$ & $100 \%$ & $100 \%$ \\
\hline Chimerism (Myeloid) & & $100 \%$ & $100 \%$ & $100 \%$ & $100 \%$ \\
\hline TCR Vb spectratyping & & & & Majority oligoclonal & \\
\hline
\end{tabular}

${ }^{* * \star}$, Count per $10^{6}$ CD3 cells; ${ }^{\wedge}$, Unenriched whole blood.

bacteremia with a central line and rhinovirus. In contrast to his brother, his engraftment is $100 \%$ across all lineages, with normal $\mathrm{T}$ cell numbers, normal TREC counts and normal proliferative response to PHA at 2 years post HCT. He has a normal ANC without need for G-CSF (Table 3). He did experience initial rituximab-induced $\mathrm{B}$ cell aplasia and reduced IgA and IgM production, but this has recently improved with normal IgA and IgM at the 24 month post HCT time point.

Patent 3 corresponds to Patient 4 of the initial published cohort of patients with X-MAID (1) (Table 4). He presented with an early onset and persistent lymphopenia and fluctuating neutropenia which responded to G-CSF treatment discovered during the exploration of an ulcerating ecthyma. Hemizygous variant in MSN gene (c.511 C>T) was found by targeted Sanger sequencing. $\mathrm{T}$ cells responded poorly to PHA stimulation (Table 4). At 1 year of age, he developed varicella requiring hospitalization and treatment by Varicella Zoster Immunoglobulin (VZIg) and acyclovir. Due to low $\mathrm{T}$ cell counts, at 5 years of age he received a matched related HCT from his geno-identical sister utilizing bone marrow $\left(3.8 \times 10^{8} \mathrm{WBC} / \mathrm{kg} ; 3.8 \times 10^{6} \mathrm{CD} 34+\right.$ cells $/ \mathrm{kg}, 29.6 \times 10^{6}$ $\mathrm{CD} 3+$ cells $/ \mathrm{kg}$ ). At that time he was in good general health, with no infectious problem. A myeloablative conditioning regimen was used (Busulfan $20 \mathrm{mg} / \mathrm{kg} /$ Cyclophosphamide $200 \mathrm{mg} / \mathrm{kg}$ ) and Cyclosporine as prophylactic treatment of GVHD. He developed skin grade 2 acute GVHD at day 10 post HCT that was resolved by cyclosporine and prednisone $(5 \mathrm{mg} / \mathrm{kg} /$ day $)$ and that recurred in ahepatic and digestive
TABLE 4 | Laboratory values for patient 3 before and after matched sibling HCT.

\begin{tabular}{|c|c|c|c|c|}
\hline & Pre-HCT & Pre-HCT & $\begin{array}{l}2 \text { months } \\
\text { post HCT }\end{array}$ & $\begin{array}{c}15 \text { years post } \\
\text { HCT }\end{array}$ \\
\hline $\begin{array}{l}\text { Age of the patient at } \\
\text { analysis }\end{array}$ & 12 months & 56 months & 65 months & 20 years \\
\hline ANC/uL & 949 & 200 & 7,500 & 4,850 \\
\hline ALC/UL & 586 & 700 & 400 & \\
\hline $\mathrm{CD} 3+/ \mathrm{ul}$ & & 658 & 276 & 1,548 \\
\hline CD4+/uL & & 203 & 60 & 720 \\
\hline CD8+/uL & & 469 & 172 & 285 \\
\hline CD4+/CD45RA+ (\%) & & 33 & & \\
\hline CD4+/CD45RO+ (\%) & & 86 & & \\
\hline $\begin{array}{l}\text { Mitogens (PHA) \% of } \\
\text { control CPM }\end{array}$ & & $11 \%$ & & \\
\hline CD19+B cells/uL & & 7 & 0 & \\
\hline NK cells/uL & & 56 & Not tested & \\
\hline Chimerism $^{\wedge \wedge}$ & & & $100 \%$ & $\begin{array}{c}100 \% \text { @ } 6 \text { years } \\
\text { post HCT }\end{array}$ \\
\hline
\end{tabular}

$\wedge$, Unenriched whole blood.

moderate form before complete resolution with corticotherapy and cyclosporine. He experienced acute pancreatitis day 26 post HCT, which was treated with octreotide, morphine, and tiemonium methysulphate. $\mathrm{He}$ experienced Varicella zoster 3 months post HCT treated by acyclovir. Chimerism has consistently been $100 \%$ of donor. $\mathrm{He}$ is in complete 
remission of his disease, with normal numbers of neutrophils and lymphocytes.

\section{DISCUSSION}

Subsequent to the identification of the proband's pathogenic variant, 7 male patients were described with moesin-deficient (XMAID), with 6 of 7 having the same MSN variant as noted in our family (1). While all of these patients demonstrated reduced $\mathrm{T}$ and $\mathrm{B}$ cells as well and hypogammaglobulinemia, there was significant variability in the initial clinical presentation. Of the 7 patients, 6 presented with Varicella in childhood, causing pneumonitis in 3 patients. Skin manifestations were common with 5 of the 7 noted to have eczema and 3 of the 7 with severe Molluscum contagiosum. Others presented with recurrent GI, GU, and respiratory infections. Six out of seven patients have been managed with prophylactic antibiotic treatment and immunoglobulin replacement and, in one case, chronic G-CSF for neutropenia. There have been two additional cases of $\mathrm{X}$ MAID published $(9,10)$. In one of these, similar to ours, profound lymphopenia was detected in follow up testing to an abnormal SCID NBS (9). This then led to WES and detection of the same p. R171W variant at 25 months of age. However, in contrast to our cases, this patient's mitogen proliferation response to PHA was robust, his clinical infection history was mild to age 2 years, and therefore he was managed supportively rather than with HCT. Of note this child was also noted to have significant eczema, a feature that has been described in other diseases of cytoskeletal regulation. Another group described detection of X-MAID due to the p. R171W variant via WES performed in a young adult man who had experienced lifelong signs and symptoms of combined immunodeficiency, including lymphopenia and neutropenia with recurrent Varicella and bacterial infections in early childhood (10). This patient had experienced clinical improvement in stability with a decrease in infection frequency following initiation of G-CSF and immunoglobulin replacement as supportive therapies. The growing body of literature describing patients with the same variants in $M S N$ gene reveals variable clinical manifestations. The reason for this is not yet elucidated, but, as previously described for other genetic forms of SCID, environmental factors could be involved in the phenotypic heterogeneity (11).

We present these three cases to demonstrate that X-MAID patients detected by either Sanger sequencing (for the oldest one) or via TREC-based newborn screening programs for SCID can present in infancy with a phenotype of T-B-NK+ SCID which benefits from correction with HCT. This represents an

\section{REFERENCES}

1. Lagresle-Peyrou C, Luce S, Ouchani F, Soheili TS, Sadek H, Chouteau M, et al. $\mathrm{X}$-linked primary immunodeficiency associated with hemizygous mutations in the MSN gene. JACI. (2016) 138:1681-9. doi: 10.1016/j.jaci.2016.04.032

2. Burns SO, Zarafov A, Thrasher AJ. Primary immunodeficiencies due to abnormalities of the actin cytoskeleton. Curr Opin Hematol. (2017) 24:16-22. doi: 10.1097/MOH.0000000000000296 additional non-radiosensitive form of T-B- NK+ SCID caused by $\mathrm{X}$-linked inheritance. While this is a limited report, the use of a fully matched cord or genoidentical donor and myeloablative conditioning was associated with myeloid engraftment and more robust immune reconstitution.

\section{CONCLUSION}

In this presentation of $3 \mathrm{X}$-MAID patients presenting with a SCID phenotype, HCT with myeloablative conditioning and utilizing a fully matched blood donors led to a better outcome than MMRD with reduced intensity conditioning.

X-MAID patients appear to have a spectrum of clinical severity despite the presence of identical variants. The reason for this is unclear, and this should be studied in greater depth through international registry collaboration with detailed immunoprofiling and mechanistic studies.

\section{DATA AVAILABILITY}

All relevant data is contained within the manuscript.

\section{ETHICS STATEMENT}

This study was carried out in accordance with the recommendations of the CHOP IRB with written informed consent from all subjects. All subjects gave written informed consent in accordance with the Declaration of Helsinki. The protocol was approved by the CHOP IRB.

\section{AUTHOR CONTRIBUTIONS}

$\mathrm{SH}$ and $\mathrm{JH}$ wrote the paper. All authors reviewed and edited the paper. IA-S and CL-P shared data on X-MAID patients prior to publication of their case series to confirm the effect of the familial mutation on MSN function. SH, MD, HJ, BN, NB, and JH cared for the patients clinically.

\section{FUNDING}

SH was supported by T32-HD043021, K12HD043245, and Clinical Immunology Society and AAAAI Foundation.

\section{ACKNOWLEDGMENTS}

Marina Cavazzana provided clinical care to patient p3. 
Wiskott-Aldrich syndrome treated by hematopoietic cell transplantation in the period 1980-2009: an international collaborative study. Blood. (2011) 118:1675-84. doi: 10.1182/blood-2010-11-319376

6. Pai SY, Logan BR, Griffith LM, Buckley RH, Parrott RE, Dvorak CC, et al. Transplantation outcomes for Severe Combined Immunodeficiency, 2000-2009. NEJM. (2014) 371:434-46. doi: 10.1056/NEJMoa14 01177

7. Kwan A, Abraham RS, Currier R, Brower A, Andruszewski K, Abbott JK, et al. Newborn screening for severe combined Immunodeficiency in 11 screening programs in the United States. JAMA. (2014) 312:729-38. doi: 10.1001/jama.2014.9132

8. Dvorak CC, Cowan MJ. Radiosensitive severe combined immunodeficiency disease. Immunol Allergy Clin North Am. (2010) 30:125-42. doi: 10.1016/j.iac.2009.10.004

9. Delmonte OM, Biggs CM, Hayward A, Comeau AM, Kuehn HS, Rosenzweig SD, et al. First case of $x$-linked moesin deficiency identified after newborn screening for SCID. J Clin Immunol. (2017) 37:336-8. doi: 10.1007/s10875-017-0391-9

10. Bradshaw G, Lualhati RR, Albury CL, Maksemous N, Roos-Araujo D, Smith RA, et al. Exome sequencing diagnoses $\mathrm{x}$-linked moesin-associated immunodeficiency in a primary immunodeficiency case. Front Immunol. (2018) 9:420. doi: 10.3389/fimmu.2018.00420

11. Notarangelo LD, Kim MS, Walter JE, Lee YN. Human RAG mutations: biochemistry and clinical implications. Nat Rev Immunol. (2016) 16:234-46. doi: $10.1038 /$ nri.2016.28

Conflict of Interest Statement: SH participated in two ad hoc advisory boards for Horizon Pharma in 2017.

The remaining authors declare that the research was conducted in the absence of any commercial or financial relationships that could be construed as a potential conflict of interest.

Copyright (c) 2019 Henrickson, Andre-Schmutz, Lagresle-Peyrou, Deardorff, Jyonouchi, Neven, Bunin and Heimall. This is an open-access article distributed under the terms of the Creative Commons Attribution License (CC BY). The use, distribution or reproduction in other forums is permitted, provided the original author(s) and the copyright owner(s) are credited and that the original publication in this journal is cited, in accordance with accepted academic practice. No use, distribution or reproduction is permitted which does not comply with these terms. 